\title{
Spectral properties of the connectivity matrix and the SIS-epidemic threshold for mid-size metapopulations
}

\author{
D. Juher ${ }^{a 1}$ and V. Mañosa ${ }^{b}$ \\ ${ }^{a}$ Dept. Informàtica, Matemàtica Aplicada i Estadística, Universitat de Girona, 17071, Girona, Spain \\ ${ }^{b}$ Dept. Matemàtica Aplicada III, Universitat Politècnica de Catalunya, 08222, Terrassa, Spain
}

\begin{abstract}
We consider the spread of an infectious disease on a heterogeneous metapopulation defined by any (correlated or uncorrelated) network. The infection evolves under transmission, recovering and migration mechanisms. We study some spectral properties of a connectivity matrix arising from the continuous-time equations of the model. In particular we show that the classical sufficient condition of instability for the disease-free equilibrium, well known for the particular case of uncorrelated networks, works also for the general case. We give also an alternative condition that yields a more accurate estimation of the epidemic threshold for correlated (either assortative or dissortative) networks.
\end{abstract}

Key words: SIS epidemic, complex networks

AMS subject classification: 05C82, 60J28, 37N25

\section{Introduction}

While the classical Susceptible-Infected-Removed (SIR) and Susceptible-Infected-Susceptible (SIS) epidemic models were based on the assumption of completely well-mixed populations, recent works have revealed the importance of considering some contact structure among populations at a geographical level (heterogeneous metapopulations). See for instance [8, 10, 11, 14, 16, 18, 21].

More precisely, one considers ensembles of local populations with a complex spatial arrangement which are pairwise connected by migration flows. So the formalism of complex networks arises $[5,6,7,15,19,22,23]$. In this paper we will deal with the following situation: the whole population is distributed inside a network of $N$ nodes and $L$ non-directed links. Without loss of

\footnotetext{
${ }^{1}$ Corresponding author. E-mail: david.juher@udg.edu
} 
generality we will assume that this network is connected, so that there is at least one path connecting any pair of nodes. Each node (or "patch") can be thought as a local population containing two types of individuals: $S$ (susceptible) and $I$ (infected). Within each patch, transmission and recovery occur between individuals of different type. Simultaneously, migratory flows take place among linked patches at constant rates.

In order to pose the continuous-time equations governing the dynamics of the corresponding SIS model it is enough to characterize the network in terms of the following two probability distributions: $p(k)$, defined as the probability that a randomly chosen node has degree $k$, and $P\left(k \mid k^{\prime}\right)$, defined as the conditional probability that a link has an end of degree $k$ when the opposite end is known to have degree $k^{\prime}$. At this point we recall the well known consistency condition [4]:

$$
k P\left(k^{\prime} \mid k\right) p(k)=k^{\prime} P\left(k \mid k^{\prime}\right) p\left(k^{\prime}\right) .
$$

It has been shown [22] that, for a suitable continuous-time formulation of the equations for the spread of infectious diseases in metapopulations, the transmission, recovery and migration processes have to take place simultaneously. Also, as usual $[6,7]$, the description of the epidemic spread is made in terms of the average number of susceptible and infected individuals per node of degree $k$ at time $t$, which here will be respectively denoted by $\rho_{S, k}(t)$ and $\rho_{I, k}(t)$. At this point let us introduce the following notation. Let $\rho_{k}(t):=\rho_{S, k}(t)+\rho_{I, k}(t)$ be the mean population per node of degree $k$. Also, for $i=S, I$, let $\rho_{i}(t)$ be the average number, at time $t$, of individuals of type $i$ per node. That is,

$$
\rho_{i}(t):=\sum_{k} p(k) \rho_{i, k}(t)
$$

Thus the mean population per node at time $t$ is $\rho(t):=\rho_{S}(t)+\rho_{I}(t)$.

The spread of the infection within a local population takes place under the assumption of a homogeneous mixing and evolves under the rules of the classical SIS model

$$
\begin{array}{r}
I \stackrel{\mu}{\longrightarrow} S \\
I+S \stackrel{\beta}{\longrightarrow} 2 I
\end{array}
$$

Here $\mu \geq 0$ is the recovery rate and $\beta \geq 0$ is the transmission rate across an infective contact. At the same time, migration of individuals $S$ and $I$ occurs between linked patches with constant coefficients $D_{S} \geq 0$ and $D_{I} \geq 0$ respectively. We assume that each migrant individual moving from a node of degree $k$ randomly chooses one of the $k$ links departing from the patch.

Now we are ready to explain the aim of this paper. Following [22] (see also [15]), in Section 2. we will derive the continuous-time equations governing the dynamics of the corresponding disease propagation. We will not assume a particular functional form for the number of contacts randomly established by each susceptible individual among the local population. Instead, we will deal with a general positive, non-decreasing function $C(x)$ which can potentially be any of the classical mass-action laws of infection [17, 24]. Once the equations are posed, we will see that a natural connectivity matrix arises from them. This matrix, denoted by $\mathcal{C}$, is directly related to the statistical characterization of the network. More precisely, $\mathcal{C}$ is defined in such a way that the entry placed at row $k$, column $k^{\prime}$ is $\mathcal{C}_{k k^{\prime}}=P\left(k^{\prime} \mid k\right) k / k^{\prime}$. In the same section we show that there exists a 
unique disease-free equilibrium. This is a well known fact for some particular forms of $C(x)$ (see Theorem 2 of [23]) and the proof can be trivially extended to the general case. In Section 3. we study some spectral properties of the connectivity matrix. Essentially we prove that $\mathcal{C}$ is similar to the stochastic matrix given by the conditional probabilities $P(i \mid j)$, and that generically all its eigenvalues are real and contained in $(-1,1]$. We also discuss the primitivity of $\mathcal{C}$ in terms of some combinatorial features of the network. In Section 4. we prove that all the eigenvalues of the Jacobian matrix at the disease-free equilibrium are real. We also get a condition for the disease-free equilibrium to become unstable. This condition reads as (Theorem 13)

$$
C\left(\frac{\rho^{0} k_{\max }}{\langle k\rangle}\right)>\frac{\mu+D_{I}}{\beta},
$$

where $\langle k\rangle=\sum_{k} k p(k)$ is the mean degree of the network, $k_{\text {max }}$ is the maximum degree of a node and $\rho^{0}$ is the initial mean population per node. It is worth noting that this result extends a well known estimation of the epidemic threshold previously obtained in the particular case of uncorrelated networks (Corollary 9 of [23]). As an example, taking $C(x):=x^{\alpha}$ for $0<\alpha \leq 1$ (a generalized unlimited or density-dependent transmission) the inequality can be read as

$$
\beta>\left(\frac{\langle k\rangle}{\rho^{0} k_{\max }}\right)^{\alpha}\left(\mu+D_{I}\right)
$$

so giving an estimate of the critical epidemic transmissibility $\beta_{c}$. Observe that for very big networks the quotient $\langle k\rangle / \rho^{0} k_{\max }$ is small. So this condition implies, in practice, the lack of an epidemic threshold for large networks with a fixed mean degree and nodes with large enough maximum connectivity. In contrast, this is not the case for mid-size networks (with, say, several hundreds of nodes). For such objects it makes sense to investigate the goodness of the previous estimation. Observe that the inequality (1.3) does not take into account the degree correlations, in the sense that it does not depend on the conditional probabilities $P\left(k \mid k^{\prime}\right)$. Since the degree correlations are expected to play a central role in the disease propagation, the estimate cannot be good in general. We prove (Theorem 14) a slight refinement of the previous estimation. It states that a condition for the disease-free equilibrium to become unstable is that, for some degree $k$,

$$
C\left(\frac{\rho^{0} k}{\langle k\rangle}\right)>\frac{\mu+D_{I}(1-P(k \mid k))}{\beta} .
$$

In Section 5. we compare the bounds of $\beta_{c}$ obtained respectively from Theorems 13 and 14 for several networks with a fixed degree distribution when the Pearson correlation coefficient ranges from values close to -1 (dissortative network) to values close to 1 (assortative network). In each case we also compute numerically the theoretical critical value of $\beta_{c}$, showing that for highly correlated networks the estimate given by Theorem 14 is much better.

\section{General equations and the disease-free equilibrium}

Consider a local population of $S$ susceptible and $I$ infected individuals. Set $x=S+I$. The number of potentially infective contacts is given by $C \times S \times \frac{I}{x}$, where $C$ is the number of contacts 
randomly established by each susceptible individual among the whole local population and $I / x$ is the probability that one of such contacts is of type $S \leftrightarrow I$. We will assume that in general $C$ is not a constant, but can take several functional forms $C(x)$ according to any of the classical mass-action laws of infection [17, 24]. Among them, $C(x)=1$ (limited or frequency-dependent transmission), $C(x)=a x^{\alpha}$ with $a>0$ and $0<\alpha \leq 1$ (a generalized unlimited or density-dependent transmission $[1,17])$, or $C(x)=a x /(b+x)$ with $a, b>0$ (saturating contact rate transmission [9]). Wherever possible, we will work with a general positive, non-decreasing function $C(x)$.

According to the derivation in [22] of the continuous-time formulation for the progress of diseases on metapopulations, the equations governing the dynamics of the disease propagation are

$$
\begin{aligned}
& \frac{d \rho_{S, k}}{d t}=\rho_{I, k}\left(\mu-\beta C\left(\rho_{k}\right) \frac{\rho_{S, k}}{\rho_{k}}\right)-D_{S} \rho_{S, k}(1-P(k \mid k))+k D_{S} \sum_{k^{\prime} \neq k} P\left(k^{\prime} \mid k\right) \frac{1}{k^{\prime}} \rho_{S, k^{\prime}} \\
& \frac{d \rho_{I, k}}{d t}=\rho_{I, k}\left(\beta C\left(\rho_{k}\right) \frac{\rho_{S, k}}{\rho_{k}}-\mu\right)-D_{I} \rho_{I, k}(1-P(k \mid k))+k D_{I} \sum_{k^{\prime} \neq k} P\left(k^{\prime} \mid k\right) \frac{1}{k^{\prime}} \rho_{I, k^{\prime}}
\end{aligned}
$$

which can be rearranged as

$$
\begin{aligned}
& \frac{d \rho_{S, k}}{d t}=\rho_{I, k}\left(\mu-\beta C\left(\rho_{k}\right) \frac{\rho_{S, k}}{\rho_{k}}\right)-D_{S} \rho_{S, k}+k D_{S} \sum_{k^{\prime}} P\left(k^{\prime} \mid k\right) \frac{1}{k^{\prime}} \rho_{S, k^{\prime}} \\
& \frac{d \rho_{I, k}}{d t}=\rho_{I, k}\left(\beta C\left(\rho_{k}\right) \frac{\rho_{S, k}}{\rho_{k}}-\mu\right)-D_{I} \rho_{I, k}+k D_{I} \sum_{k^{\prime}} P\left(k^{\prime} \mid k\right) \frac{1}{k^{\prime}} \rho_{I, k^{\prime}}
\end{aligned}
$$

where, for the sake of simplicity, we have dropped the time argument from the the equations. Observe that we have a system of $2 n$ differential equations, where $n$ is the number of degrees present in the network. From now on we will use the following standing notation. The degrees present in the network will be denoted by

$$
k_{\text {min }}=k_{1}<k_{2}<\ldots<k_{n}=k_{\max } .
$$

At this point it is convenient to perform a change of notation to have the state variables of the equations indexed from 1 to $n$. Set $S_{i}:=\rho_{S, k_{i}}$ and $I_{i}:=\rho_{I, k_{i}}$ for $1 \leq i \leq n$. With this notation, the previous equations read as

$$
\begin{aligned}
\frac{d}{d t} S_{i}(t) & =I_{i}\left(\mu-\beta C\left(S_{i}+I_{i}\right) \frac{S_{i}}{S_{i}+I_{i}}\right)-D_{S} S_{i}+k_{i} D_{S} \sum_{j} P\left(k_{j} \mid k_{i}\right) \frac{1}{k_{j}} S_{j} \\
\frac{d}{d t} I_{i}(t) & =I_{i}\left(\beta C\left(S_{i}+I_{i}\right) \frac{S_{i}}{S_{i}+I_{i}}-\mu\right)-D_{I} I_{i}+k_{i} D_{I} \sum_{j} P\left(k_{j} \mid k_{i}\right) \frac{1}{k_{j}} I_{j}
\end{aligned}
$$

We recall [15] that multiplying equations (2.3) and (2.4) by $p\left(k_{i}\right)$, summing over all $i$, and using (1.1) yields that the mean population per node $\rho(t)$ remains constant. From now on, this constant will be denoted by $\rho^{0}$. 
For any matrix $M$, the entry placed at row $i$, column $j$ will be denoted by $M_{i j}$. Following [15], we define the elements of the connectivity matrix $\mathcal{C}$ as

$$
\mathcal{C}_{i j}=\frac{k_{i}}{k_{j}} P\left(k_{j} \mid k_{i}\right)
$$

Observe that $\mathcal{C}$ is an $n \times n$ nonnegative matrix. We recall that a square matrix $M$ with nonnegative entries is called reducible if there exists a permutation matrix $P$ such that

$$
P^{-1} M P=\left(\begin{array}{cc}
M_{11} & 0 \\
M_{21} & M_{22}
\end{array}\right)
$$

where $M_{11}$ and $M_{22}$ are square matrices of sizes $l \times l$ and $m \times m(l, m \geq 1)$ respectively and 0 stands for the $l \times m$ matrix whose entries are all 0 . If there does not exist such $P$ then the matrix $M$ is called irreducible.

The spectral radius of a square matrix $M$ will be denoted by $\sigma(M)$. We recall that it is defined as the maximum of the moduli of the eigenvalues of $M$.

The following result states some basic properties of the connectivity matrix.

Lemma 1. Let $\mathcal{C}$ be the connectivity matrix. Then:

a) $\mathcal{C}$ is irreducible.

b) The vector $v=\left(k_{1}, \ldots, k_{n}\right)$ is an eigenvector of $\mathcal{C}$ of eigenvalue 1.

c) $\sigma(\mathcal{C})=1, \sigma(\mathcal{C})$ is a simple eigenvalue of $\mathcal{C}$, and any nonnegative eigenvector of $\mathcal{C}$ is a multiple of $v$.

Proof. To prove (a), assume that $\mathcal{C}$ is reducible, so that $\mathcal{C}$ can be written as

$$
\left(\begin{array}{cc}
\mathcal{C}_{11} & 0 \\
\mathcal{C}_{21} & \mathcal{C}_{22}
\end{array}\right)
$$

for some square matrices $\mathcal{C}_{11}$ and $\mathcal{C}_{22}$ of sizes $l \times l$ and $m \times m(l, m \geq 1)$ respectively. This means that there exists a proper subset $X=\left\{j_{1}, \ldots, j_{l}\right\}$ of degrees such that $P\left(k^{\prime} \mid k\right)=0$ for any $k^{\prime} \notin X$. In consequence, the network is disconnected, in contradiction with the hypotheses. To prove (b) observe that the $i$-th entry of $\mathcal{C} \cdot v$ is

$$
\sum_{j=1}^{n} \mathcal{C}_{i j} k_{j}=k_{i} \sum_{j=1}^{n} P\left(k_{j} \mid k_{i}\right)=k_{i}
$$

Finally, (c) is a consequence of (a), (b) and the Perron-Frobenius Theorem (see Theorem 8.4.4 of [13] for instance).

Lemma 1(c) can be used to prove that the system of differential equations given by (2.3) and (2.4) has a unique disease-free equilibrium, that is, a steady state $\left\{S_{i}^{\star}, I_{i}^{\star}\right\}$ for which $I_{i}^{\star}=0$ and $S_{i}^{\star}>0$ for all $1 \leq i \leq n$. The result is well known for some particular classes of transmission (see, for instance, Theorem 2 of [23]) and the proof can be straightforwardly extended to our general setting. 
Theorem 2. There is only one disease-free equilibrium of the equations (2.3) and (2.4) and it is given by $S_{i}^{\star}=\rho^{0} k_{i} /\langle k\rangle, I_{i}^{\star}=0$ for $1 \leq i \leq n$, where $\langle k\rangle=\sum_{k} k p(k)$ is the mean degree of the network.

Proof. Setting $\frac{d}{d t} S_{i}(t)=\frac{d}{d t} I_{i}(t)=I_{i}^{\star}=0$ yields

$$
S_{i}^{\star}=\sum_{j} \mathcal{C}_{i j} S_{j}^{\star}
$$

A solution for this equation has to be an eigenvector of $\mathcal{C}$ of eigenvalue 1. By Lemma 1(c), it has to be $S_{i}^{\star}=\alpha k_{i}$ for some $\alpha$ and all $i$. To determine $\alpha$, simply observe that

$$
\rho^{0}=\sum_{i} p\left(k_{i}\right) S_{i}^{\star}=\sum_{i} p\left(k_{i}\right) \alpha k_{i}=\alpha\langle k\rangle
$$

\section{The spectrum of the connectivity matrix}

In this section we look closer at the spectrum of the connectivity matrix. We start by proving that $\mathcal{C}$ is similar to the stochastic matrix defined by the conditional probability $P(i \mid j)$. From now on, $\operatorname{diag}\left(a_{1}, a_{2}, \ldots, a_{n}\right)$ will stand for a diagonal matrix $D$ such that $D_{i i}=a_{i}$. A nonnegative square matrix will be called row (column) stochastic if each row (column) sums to 1 . The spectrum of a square matrix $M$ will be denoted by $\operatorname{sp}(M)$. The transpose of $M$ will be denoted by $M^{T}$. From now on, $P$ will denote the $n \times n$ matrix such that $P_{i j}=P\left(k_{j} \mid k_{i}\right)$. Observe that $P$ is row stochastic (so, $P^{T}$ is column stochastic). We also recall that the spectral radius of any (row or column) stochastic matrix is 1 and that it corresponds to an eigenvalue.

Lemma 3. Let $A=\operatorname{diag}\left(k_{1}, \ldots, k_{n}\right)$. Then, $A^{-1} \mathcal{C} A=P$.

Proof. Set $X:=\mathcal{C} A$. Then, $X_{i j}=\sum_{m=1}^{n} \mathcal{C}_{i m} A_{m j}=\mathcal{C}_{i j} A_{j j}=k_{i} P\left(k_{j} \mid k_{i}\right)$. Therefore,

$$
\left(A^{-1} \mathcal{C} A\right)_{i j}=\sum_{m=1}^{n}\left(A^{-1}\right)_{i m} X_{m j}=\left(A^{-1}\right)_{i i} X_{i j}=\frac{1}{k_{i}} k_{i} P\left(k_{j} \mid k_{i}\right)=P\left(k_{j} \mid k_{i}\right)=P_{i j} .
$$

Corollary 4. $\operatorname{sp}(\mathcal{C})=\operatorname{sp}(P)=\operatorname{sp}\left(P^{T}\right)$. In particular, 1 is a simple maximal eigenvalue of $\mathcal{C}$.

Proof. The equalities of the spectra follow from Lemma 3. The fact that 1 is a simple eigenvalue was stated in Lemma 1(c).

In principle, $\operatorname{sp}(\mathcal{C})$ could contain complex eigenvalues. Next we will prove that $P^{T}$ is similar to a real $n \times n$ symmetric matrix. From this fact and Corollary 4 we get that the spectrum of $\mathcal{C}$ is real and contained in $[-1,1]$ (Proposition 6).

Lemma 5. Let $B=\operatorname{diag}\left(v_{1}, \ldots, v_{n}\right)$, with $v_{i}=\sqrt{k_{i} p\left(k_{i}\right)}$. Then, $B^{-1} P^{T} B$ is symmetric. 
Proof. Let $X=B^{-1} P^{T}$ and $Z=X B$. Then,

$$
X_{i j}=\sum_{l=1}^{n}\left(B^{-1}\right)_{i l}\left(P^{T}\right)_{l j}=\left(B^{-1}\right)_{i i}\left(P^{T}\right)_{i j}=\frac{P\left(k_{i} \mid k_{j}\right)}{\sqrt{k_{i} p\left(k_{i}\right)}} .
$$

Therefore,

$$
Z_{i j}=\sum_{l=1}^{n} X_{i l} B_{l j}=X_{i j} B_{j j}=P\left(k_{i} \mid k_{j}\right) \sqrt{\frac{k_{j} p\left(k_{j}\right)}{k_{i} p\left(k_{i}\right)}} .
$$

Now, a straightforward computation using (1.1) shows that $Z_{i j}=Z_{j i}$.

As an immediate corollary of the above results we obtain

Proposition 6. All the eigenvalues of $\mathcal{C}$ are real and contained in $[-1,1]$. In particular, 1 is a simple maximal eigenvalue of $\mathcal{C}$.

We recall that a square nonnegative matrix $M$ is called primitive if and only if there exists $m \geq 1$ such that all entries of $M^{m}$ are positive.

Remark 7. From the definition of the connectivity matrix it immediately follows that $\mathcal{C}$ is primitive if and only if $P$ is primitive (equivalently, if and only if $P^{T}$ is primitive).

The statement of Proposition 6 can be slightly improved by adding the extra hypothesis of primitivity on $\mathcal{C}$. This is a consequence of a well known fact (see for instance Theorem 1.7 of [3]): for any primitive matrix $M, \sigma(M)$ is greater in magnitude than any other eigenvalue. Thus we get the following result:

Proposition 8. If $\mathcal{C}$ is primitive, then all the eigenvalues of $\mathcal{C}$ are real and can be labeled in such a way that $-1<\lambda_{1} \leq \lambda_{2} \leq \ldots \leq \lambda_{n-1}<\lambda_{n}=1$.

In fact, the primitivity condition in Proposition 8 is not restrictive. To see it, let us recall some simple and well known facts about matrices and associated graphs (see Chapter 2 of [3]). Let $M$ be an $m \times m$ nonnegative matrix. To $M$ one can associate a directed graph $G$ of $m$ vertices $v_{1}, \ldots, v_{m}$ in such a way that there is a directed link from $v_{i}$ to $v_{j}$ if and only if $M_{i j} \neq 0$. The adjacency matrix of $G$ is an $m \times m$ matrix $A$ such that $A_{i j}=1$ if there is a directed link from $v_{i}$ to $v_{j}$ and $A_{i j}=0$ otherwise. A simple circuit in $G$ is a sequence of vertices $v_{i_{0}}, \ldots, v_{i_{l}}$ such that $v_{i_{0}}=v_{i_{l}}$, there is a directed link in $G$ from $v_{i_{j}}$ to $v_{i_{j+1}}$ for $0 \leq j<l$ and $v_{i_{1}}, \ldots, v_{i_{l}}$ are all distinct. In this case, $l$ is the length of such circuit. It is well known that $M$ (equivalently, $A$ ) is primitive if and only if the greatest common divisor of the lengths of all simple circuits in $G$ is 1 (see Theorem 2.30 of [3]). In particular, $M$ (equivalently, $A$ ) is primitive if there is a diagonal entry different from 0 . Now observe that when $A$ is a symmetric matrix (equivalently, when $M_{i j}=0$ if and only if $M_{j i}=0$ ), the graph $G$ can be thought as a non-directed graph, since any pair of directed links (from $v_{i}$ to $v_{j}$ and from $v_{j}$ to $v_{i}$ ) can be thought as an undirected link joining $v_{i}$ and $v_{j}$. In this case, either some diagonal entries of $A$ are different from 0 or there are simple circuits of length 2 (take $i \neq j$ such that $A_{i j} \neq 0$ and consider the simple circuit given by the triplet $\left.v_{i}, v_{j}, v_{i}\right)$. Then, $A$ and $M$ are primitive if there are simple circuits in $G$ of odd length. Collecting it all, we have the following result. 

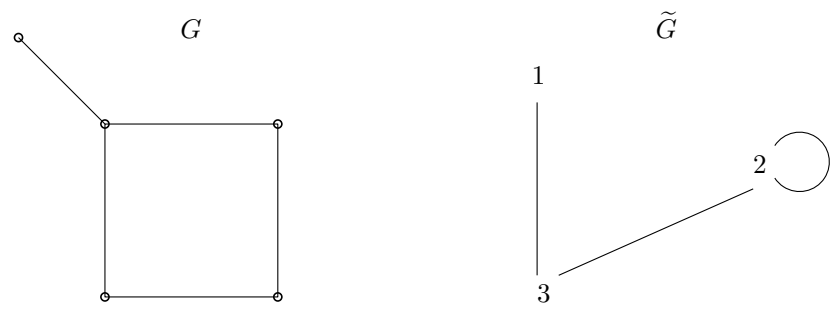

Figure 1: A graph $G$ and its corresponding degree graph $\widetilde{G}$

Lemma 9. Let $A$ be the adjacency matrix of an undirected network $G$. Then, $A$ is primitive if and only if there are simple circuits of odd length in $G$.

Now we turn back to our situation, so that $G$ is the (undirected) population network of $N$ nodes, there are $n$ different degrees $k_{1}<k_{2}<\ldots<k_{n}$ and $\mathcal{C}$ is the connectivity matrix, of size $n \times n$. To $\mathcal{C}$ we can then associate a graph $\widetilde{G}$ of $n$ nodes $\left\{k_{1}, \ldots, k_{n}\right\}$, which we call the degree network. Let $A$ be the adjacency matrix of $G$. Observe that $A$ is symmetric and that if $A_{i j} \neq 0$ then $\mathcal{C}_{k l} \neq 0$, where $k$ and $l$ are the degrees of $v_{i}$ and $v_{j}$ respectively. Conversely, if $\mathcal{C}_{k l} \neq 0$ then there exist vertices $v_{i}$ and $v_{j}$ of respective degrees $k$ and $l$ which are adjacent in $G$. Moreover, from (1.1) it follows that the adjacency matrix of $\widetilde{G}$ is symmetric (equivalently, the graph $\widetilde{G}$ is undirected). See Figure 1 for an example. We have the following result.

Theorem 10. Let $A$ be the adjacency matrix of an undirected network $G$. Let $\mathcal{C}$ be the connectivity matrix. If $A$ is primitive, then $\mathcal{C}$ is primitive.

Proof. Let $\widetilde{A}$ be the adjacency matrix of the corresponding degree network $\widetilde{G}$. To see that $\mathcal{C}$ is primitive it is obviously enough to see that $\widetilde{A}$ is primitive. Observe that if there are two adjacent nodes in $G$ with the same degree $k$, then $\widetilde{A}_{k k} \neq 0$ and, in consequence, $\widetilde{A}$ is primitive by Lemma 9 . So we are done in this case. From now on, assume that there are no adjacent nodes in $G$ with the same degree. By Lemma 9, there is a simple circuit $x_{0}, x_{1}, \ldots, x_{l}=x_{0}$ in $G$ with $l \geq 3$ odd. For $0 \leq i \leq l$, let $d_{i}$ be the degree of the node $x_{i}$ in $G$. If all $d_{i}$ are distinct, then $d_{0}, d_{1}, \ldots, d_{l}$ is a simple circuit of odd length in $\widetilde{G}$. In consequence, by Lemma $9, \widetilde{A}$ is primitive and we are done. So, let us assume that there are at least two indices $i_{1}<i_{2}<\ldots<i_{r}$ such that $d_{i_{1}}=d_{i_{2}}=\ldots=d_{i_{r}}$. Without loss of generality we can assume that $i_{1}=0$ and $i_{r} \geq l-2$. Now consider the intermediate circuits in $\widetilde{G}: d_{i_{j}}, d_{i_{j}+1}, \ldots, d_{i_{j+1}}$, for $1 \leq j<r$, together with the circuit $d_{i_{r}}, d_{i_{r}+1}, \ldots, d_{l}=d_{0}$. Observe that, since the sum of the lengths of such circuits is $l$, odd, at least one of these lengths is odd. If the corresponding circuit in $\widetilde{G}$ is simple, we are done by Lemma 9. Otherwise, we can repeat the argument starting from the corresponding circuit (whose length is strictly smaller than l).

Of course, the converse of Theorem 10 is not true. For an example, consider the graph $G$ and its corresponding degree graph $\widetilde{G}$ depicted in Figure 1. For a convenient labeling of the nodes, the 
respective adjacency matrices $A$ and $\widetilde{A}$ read as

$$
A=\left(\begin{array}{lllll}
0 & 1 & 0 & 0 & 0 \\
1 & 0 & 1 & 0 & 1 \\
0 & 1 & 0 & 1 & 0 \\
0 & 0 & 1 & 0 & 1 \\
0 & 1 & 0 & 1 & 0
\end{array}\right), \widetilde{A}=\left(\begin{array}{lll}
0 & 0 & 1 \\
0 & 1 & 1 \\
1 & 1 & 0
\end{array}\right)
$$

It is easy to check that $A$ is not primitive, while $\widetilde{A}$ (and, so, the associated connectivity matrix $\mathcal{C}$ ) is primitive because in particular $\widetilde{A}_{22} \neq 0$.

Now we are ready to discuss the primitivity condition on $\mathcal{C}$ in Proposition 8. Typically, the population network is a large graph randomly constructed from the configuration model. In other words, it is a randomized realization of the particular probability distributions $p(k)$ and $P\left(k \mid k^{\prime}\right)$. Of course, with a high probability this network will have simple circuits of odd length. In consequence, by Lemma 9 and Theorem 10, the associated connectivity matrix $\mathcal{C}$ will be primitive. Even when the obtained network does not fulfill this condition, with a certain probability there will be two adjacent nodes with the same degree $k$. In this case, independently on the primitivity of the adjacency matrix we will have $\mathcal{C}_{k k} \neq 0$ and, in consequence, $\mathcal{C}$ will also be primitive. But even in the very unlikely case in which all these conditions fail (consider for instance an exponential or a scale-free network $G$ obtained by a growing mechanism $[2,12]$ such as the addition of new nodes of degree 1 which leads to a tree, without circuits of odd length), if for some reason we need the connectivity matrix to be primitive, then we can slightly modify the network by choosing a node $v$ and attaching to it a chain of three new consecutive nodes $\left\{w_{1}, w_{2}, w_{3}\right\}$ such that $w_{1}$ and $w_{2}$ have degree 2 and $w_{3}$ has degree 1 . Now observe that the associated connectivity matrix satisfies $\mathcal{C}_{22} \neq 0$ and is, in consequence, primitive. For a large network, this minor modification does not significantly change its statistical properties and, in consequence, is not expected to change the global behaviour of the infection spread.

\section{An estimation of the epidemic threshold}

Linearizing the system of differential equations (2.3) and (2.4) at the disease-free equilibrium one gets that the associated Jacobian matrix is

$$
J=\left(\begin{array}{cc}
X & Y \\
0 & Z
\end{array}\right)
$$

where $X, Y, Z$ are $n \times n$ matrices, with $X=D_{S}(\mathcal{C}-\mathrm{Id})$ and $Z=\operatorname{diag}\left(\beta C\left(S_{i}^{\star}\right)-\mu-D_{I}\right)+D_{I} \mathcal{C}$ which, by Theorem 2 , equals $\operatorname{diag}\left(\beta C\left(\rho^{0} k_{i} /\langle k\rangle\right)-\mu-D_{I}\right)+D_{I} \mathcal{C}$. This is a standing notation for the rest of this section. We start by using the results of the previous sections to show that the spectrum of $J$ is real.

Proposition 11. All the eigenvalues of the Jacobian matrix J are real. 
Proof. By (4.1), the spectrum of $J$ equals $\operatorname{sp}(X) \cup \operatorname{sp}(Z)$. From Proposition 6 it follows that all the eigenvalues of $\mathcal{C}-\operatorname{Id}$ (in consequence, those of $X$ ) are real. Let us see that those of $Z$ are also real. Set $D:=\operatorname{diag}\left(\beta C\left(\rho^{0} k_{i} /\langle k\rangle\right)-\mu-D_{I}\right)$. Then, $Z=D+D_{I} \mathcal{C}$. Now set $A:=\operatorname{diag}\left(k_{1}, \ldots, k_{n}\right)$. Then, $A^{-1} Z A=D+D_{I} A^{-1} \mathcal{C} A$ which, by Lemma 3, equals $D+D_{I} P$. Therefore,

$$
\operatorname{sp}(Z)=\operatorname{sp}\left(D+D_{I} P\right)=\operatorname{sp}\left(D+D_{I} P^{T}\right) .
$$

Now set $B:=\operatorname{diag}\left(v_{1}, \ldots, v_{n}\right)$, with $v_{i}=\sqrt{k_{i} p\left(k_{i}\right)}$. From (4.2) it follows that

$$
\operatorname{sp}(Z)=\operatorname{sp}\left(B^{-1}\left(D+D_{I} P^{T}\right) B\right) .
$$

Now observe that $B^{-1}\left(D+D_{I} P^{T}\right) B=D+D_{I}\left(B^{-1} P^{T} B\right)$ which, by Lemma 5 , is a symmetric matrix and has, in consequence, all its eigenvalues real.

Next we look closer at the spectrum of the Jacobian matrix $J$ in order to find sufficient conditions of instability for the disease-free equilibrium. The proofs of Theorems 13 and 14 are based on the following result.

Lemma 12. Let $D=\operatorname{diag}\left(d_{1}, \ldots, d_{n}\right)$ in such a way that $d_{i}>0$ for some $i$. Let $M$ be an irreducible nonnegative matrix and let $r>0$. Then, the matrix $D+r M$ has a positive eigenvalue.

Proof. Relabel the diagonal elements of $D$ as $a_{i}$ in such a way that $a_{1} \leq a_{2} \leq \ldots \leq a_{n}$. Suppose that $a_{1}>0$. Then, $D+r M$ is an irreducible nonnegative matrix. Thus, by Perron-Frobenius Theorem, $\sigma(D+r M)>0$ is an eigenvalue of $D+r M$ and we are done.

Now assume that $a_{1}<0$. Take any $x>\left|a_{1}\right|>0$. By the same argument, $\sigma(D+x \operatorname{Id}+r M)>0$ is an eigenvalue of $D+x \operatorname{Id}+r M$. In consequence, $\lambda:=\sigma(D+x \operatorname{Id}+r M)-x$ is an eigenvalue of $D+r M$. Let us show that $\lambda>0$. Observe that $D+x \operatorname{Id}+r M \geq D+x \operatorname{Id} \geq 0$ (by abuse of notation, here the inequalities hold entrywise). In consequence, $(D+x \operatorname{Id}+r M)^{m} \geq(D+x \operatorname{Id})^{m}$ for every $m \geq 1$. Since $\sigma(X)=\lim _{m \rightarrow \infty}\|X\|^{1 / m}$ for any matrix $X$ and any entrywise norm $\|\cdot\|$, it follows that $\sigma(D+x \operatorname{Id}+r M) \geq \sigma(D+x \operatorname{Id})=a_{n}+x$. Therefore, $\lambda=\sigma(D+x \operatorname{Id}+r M)-x \geq a_{n}>0$.

Theorem 13. A sufficient condition for the disease-free equilibrium to become unstable is

$$
C\left(\frac{\rho^{0} k_{\max }}{\langle k\rangle}\right)>\frac{\mu+D_{I}}{\beta} .
$$

Proof. Recall that $\operatorname{sp}(J)=\operatorname{sp}(X) \cup \operatorname{sp}(Z)$ according to (4.1). From Proposition 6 it follows that all the eigenvalues of $\mathcal{C}-$ Id (in consequence, those of $X$ ) are negative or zero. On the other hand, since $C(x)$ is a non-decreasing function by hypothesis, it turns out that the largest diagonal element of $D:=\operatorname{diag}\left(\beta C\left(\rho^{0} k_{i} /\langle k\rangle\right)-\mu-D_{I}\right)$ is obtained by taking $k_{i}=k_{\max }$. In addition, $\mathcal{C}$ is irreducible by Lemma 1(a). Therefore, using Lemma 12 with $r=D_{I}$ and $M=\mathcal{C}$ we get that if $\beta C\left(\rho^{0} k_{\max } /\langle k\rangle\right)-\mu-D_{I}>0$ then $\operatorname{sp}(Z)$ contains a positive eigenvalue and the disease-free equilibrium is unstable.

Observe that the instability condition given by Theorem 13 is independent of the conditional probabilities $P\left(k \mid k^{\prime}\right)$. In other words, it is independent from the degree correlations. Since correlations in the contact network structure are expected to play some kind of role in the disease 
spread, the inequality in Theorem 13 cannot be an optimal estimate of the epidemic threshold in general. The following refinement of Theorem 13 can be a much better estimate in some cases (highly correlated mid-size networks, as discussed in the next section).

Theorem 14. A sufficient condition for the disease-free equilibrium to become unstable is that, for some $1 \leq i \leq n$,

$$
C\left(\frac{\rho^{0} k_{i}}{\langle k\rangle}\right)>\frac{\mu+D_{I}\left(1-P\left(k_{i} \mid k_{i}\right)\right)}{\beta} .
$$

Proof. We recover the notation used in the proof of Theorem 13. Observe that the matrix $Z$ can be also written as $\operatorname{diag}\left(\beta C\left(\rho^{0} k_{i} /\langle k\rangle\right)-\mu-D_{I}+D_{I} P\left(k_{i} \mid k_{i}\right)\right)+D_{I} \mathcal{C}^{\star}$, where $\mathcal{C}^{\star}$ is the matrix obtained from $\mathcal{C}$ replacing all its diagonal entries by 0 . Since the irreducible character of a matrix is not affected by its diagonal entries, $\mathcal{C}^{\star}$ is irreducible (and nonnegative). Thus we can use again Lemma 12 with $D=\operatorname{diag}\left(\beta C\left(\rho^{0} k_{i} /\langle k\rangle\right)-\mu-D_{I}+D_{I} P\left(k_{i} \mid k_{i}\right)\right), r=D_{I}$ and $M=\mathcal{C}^{\star}$ in order to obtain the desired condition.

Intuitively, the term $1-P\left(k_{i} \mid k_{i}\right)$ in the statement of Theorem 14 accounts for the infected individuals diffusing from patches of degree $k_{i}$ to adjacent patches of degree different from $k_{i}$, so contributing to the decrease of $I_{i}$. Observe the second term in the right hand side of equation (2.2).

\section{Simulations}

To fix ideas, we will work with the contact function $C(x)=x^{\alpha}$ for $0<\alpha \leq 1$. For every network considered in this section, we will distribute the individuals in such a way that, at time $t=0$, every node $v$ contains $k+10$ individuals, where $k$ is the degree of $v$. In consequence, $\rho^{0}=\langle k\rangle+10$. Taking it all into account, Theorem 13 implies that the disease-free equilibrium is unstable when $\beta>\beta_{0}$, where

$$
\beta_{0}=\left(\mu+D_{I}\right)\left(\frac{\langle k\rangle}{(\langle k\rangle+10) k_{\max }}\right)^{\alpha} \text {. }
$$

On the other hand, Theorem 14 tells us that the disease-free equilibrium is unstable if $\beta>\beta_{1}$, where

$$
\beta_{1}=\min _{i=1, \ldots, n}\left\{\left(\mu+D_{I}\left(1-P\left(k_{i} \mid k_{i}\right)\right)\right)\left(\frac{\langle k\rangle}{(\langle k\rangle+10) k_{i}}\right)^{\alpha}\right\} .
$$

Observe that $\beta_{0}$ depends only on the degree distribution $p(k)$, while $\beta_{1}$ depends also on the diagonal of the conditional probability matrix $P$.

To analyze the role of correlations in the estimation of the epidemic threshold given by (5.2) we have performed a sequence of experiments of the following type. We start by fixing a degree distribution $p(k)$. Then, using the classical configuration model, we randomly generate a mid-size network ( $N=500$ nodes) whose degree distribution fits to $p(k)$. We will refer to this network, which is uncorrelated in general, as $X_{0}$. To measure the correlations present in a given network $X$ we will use the classical Pearson coefficient $r$ computed from the two random variables defined by the degrees of the nodes at both ends of randomly chosen links in $X$ (see [20]). Values of $r$ close 


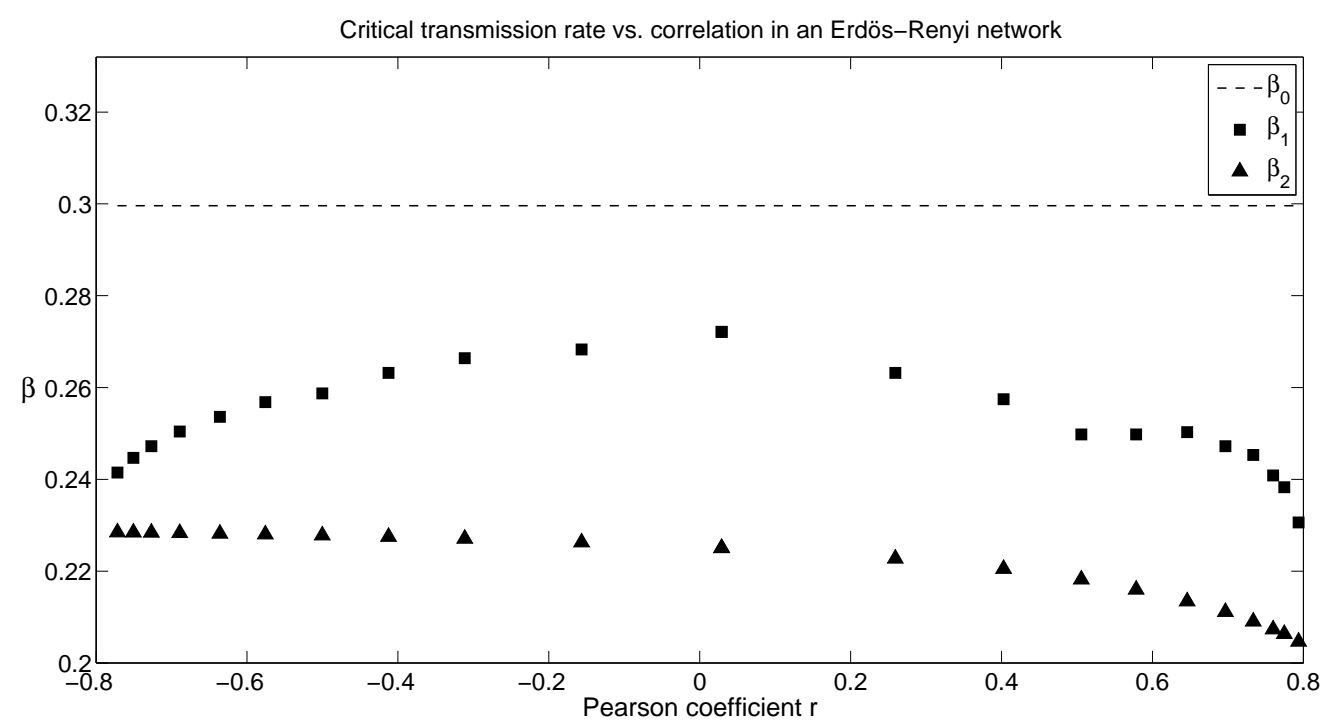

Figure 2: Critical transmission rate estimations vs. correlation in an Erdős-Renyi network, $N=$ $500,\langle k\rangle=6, \alpha=0.5, \mu=D_{I}=1$.

to -1 (respectively 1 ) account for dissortative (resp. assortative) networks, while values close to 0 correspond to uncorrelated networks. We will proceed as follows. Starting from $X_{0}$, we will use a well-known rewiring algorithm, first introduced by Xulvi-Brunet and Sokolov in [25], in order to change correlations in $X_{0}$ without modifying its degree distribution. Roughly speaking, at each step a pair of non connected links are chosen at random and their four endpoints are cross rewired, if necessary, in order that the new links connect the two nodes with the smaller degrees and the two nodes with the larger degrees. In the dissortative version of the algorithm, we rewire in such a way that one of the new links connects the node with the largest degree to the node with lowest degree. In fact we use a slightly modified version of these algorithms to assure that the obtained networks are always connected. We execute 5000 iterations of both algorithms, starting from $X_{0}$, to get correlated networks with degree distribution $p(k)$ and coefficients $r$ ranging from 0 to values close to 1 (respectively, -1 ). Of course, $|r|$ does not really attain values very close to 1 due to the finite size effect. Every 500 iterations we compute the corresponding coefficient $r$ and the constant $\beta_{1}$ as in (5.2). In addition, we compute numerically $\beta_{2}$, defined as the value of $\beta$ for which the largest eigenvalue of the lower right diagonal block $Z$ of the Jacobian matrix (4.1) is zero, so that $\beta_{2}$ can be viewed as the theoretical epidemic threshold.

The results for a particular Erdős-Renyi network with $N=500,\langle k\rangle=6, \alpha=1 / 2, \mu=$ $D_{I}=1$ and $\rho^{0}=\langle k\rangle+10$ are shown in Figure 2. For this network, the degrees $k_{i}$ range from 1 to $17, \beta_{0}=0.299605$, the coefficients $r$ range from -0.771773 to 0.793681 and $\beta_{1}$ ranges from 0.230613 to 0.272136 . The optimal critical transmissibility rates $\beta_{2}$, computed numerically from the Jacobian matrix of the disease-free equilibrium, are also shown. As can be seen the bound of the critical transmission rate given by $\beta_{1}$ improves significatively the one given by $\beta_{0}$, in particular 


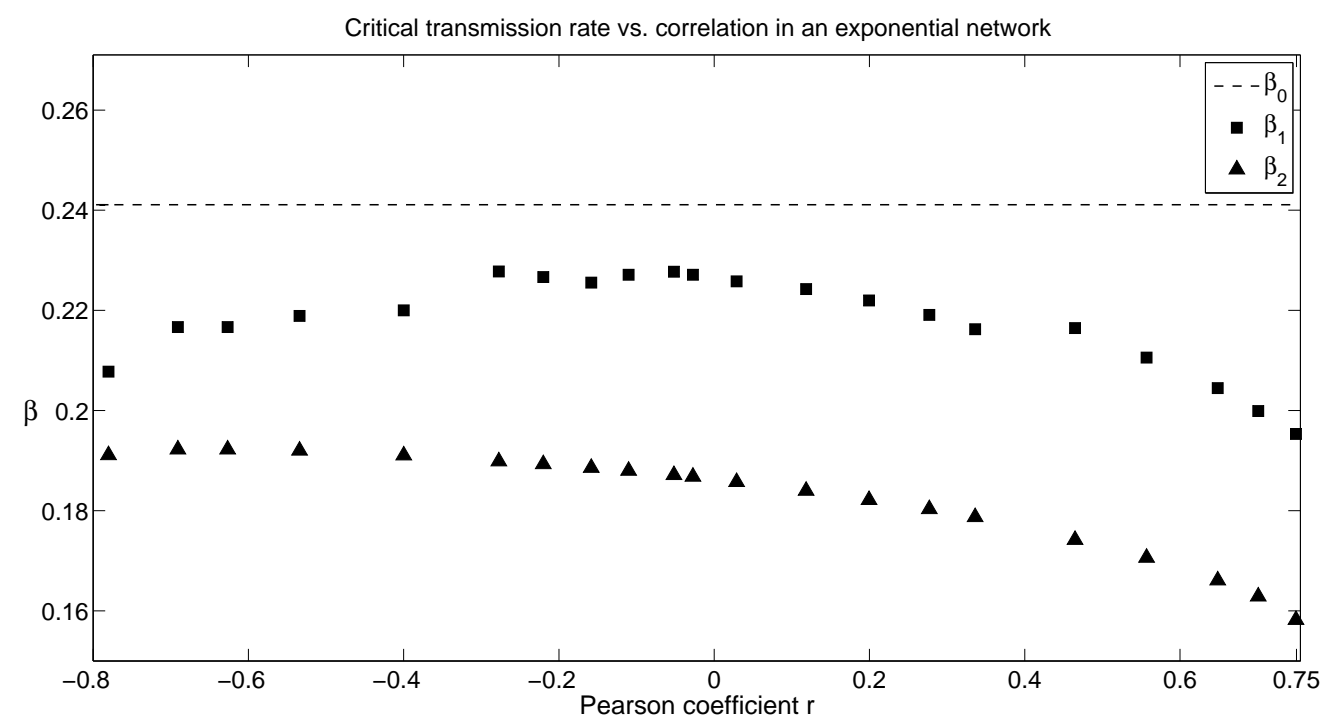

Figure 3: Critical transmission rate estimations vs. correlation in an exponential network, $N=$ $500,\langle k\rangle=4, \alpha=0.5, \mu=D_{I}=1$.

for the high correlated cases. The major improvement is obtained for dissortative networks.

The same experiment has been performed with exponential networks, that is, $p(k) \sim e^{-k /\langle k\rangle}$. In Figure 3 we have shown a particular realization of the experiment, with $N=500,\langle k\rangle=4$, $\alpha=1 / 2, \mu=D_{I}=1$ and $\rho^{0}=\langle k\rangle+10$. In this case the degrees present in the network are $\{1,2, \ldots, 19,25,26\}, \beta_{0}=0.241106$, the coefficients $r$ range from -0.7801048 to 0.749340 and $\beta_{1}$ ranges from to 0.195327 to 0.227773 . Again the bound given by $\beta_{1}$ is closer to the theoretical threshold $\beta_{2}$ in the dissortative cases.

Finally, scale-free networks of $N=500$ nodes have been generated via the classical preferential attachment mechanism [2], with $\langle k\rangle=6$. The corresponding results for one of such networks, with $\mu=1, \alpha=1 / 2, D_{I}=5$ and $\rho^{0}=\langle k\rangle+10$, are shown in Figure 4. The degrees present in the network are $\{3,4, \ldots, 18,21,23,35,36,44,46,55,58,62,63,73,79\}, \beta_{0}=0.415264$ and the coefficients $r$ range from -0.353693 to 0.087138 . Observe that the scale-free architecture enforces a major restriction on the maximum possible value of the correlation coefficient. In this case, $\beta_{1}$ ranges from 0.189496 to 0.380368 . In contrast to the previous cases, here the improvement given by $\beta_{1}$ grows very quickly even for a small increase of the assortativity.

\section{Conclusions}

We consider the spread of an infectious disease on a heterogeneous metapopulation defined by a general network. The infection evolves under transmission, recovering and migration mechanisms. We also consider that in each node the infection is modeled by the classical mass-action laws with 


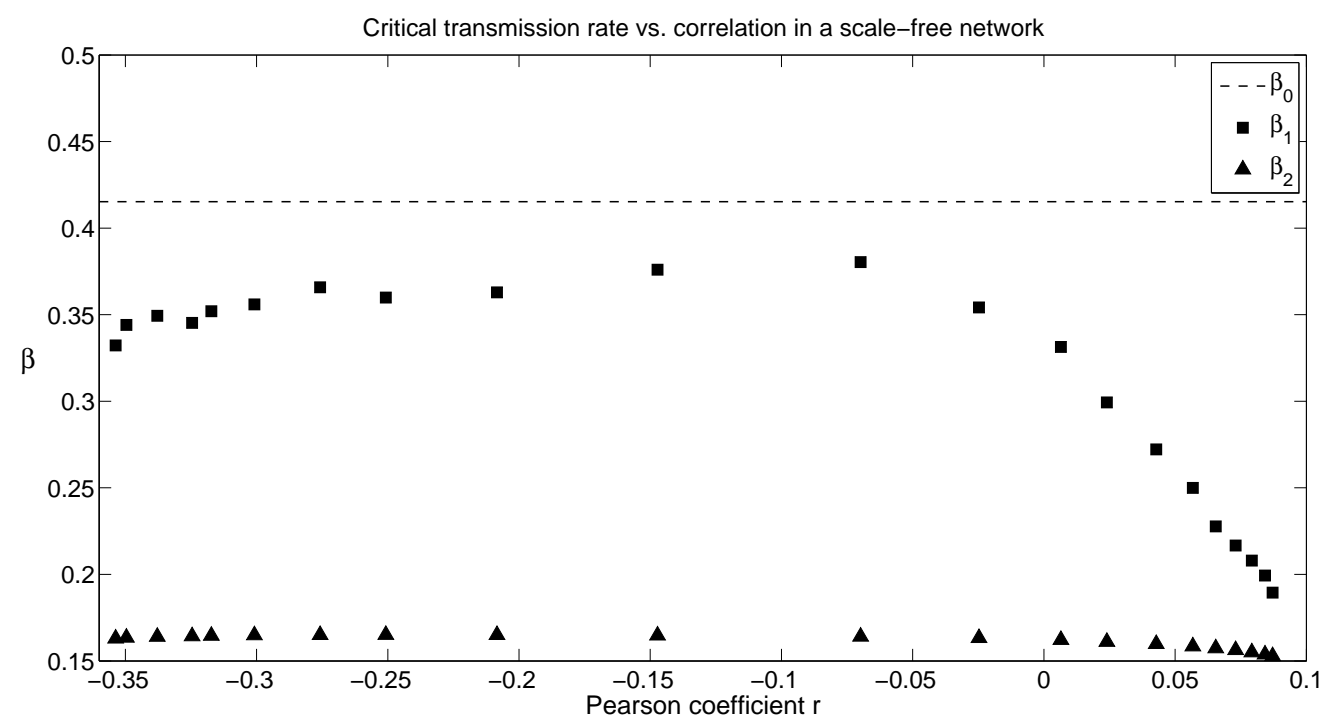

Figure 4: Critical transmission rate estimations vs. correlation in a scale-free network, $N=500$, $\langle k\rangle=6, \alpha=0.5, \mu=1, D_{I}=5$.

general positive non-decreasing contact functions. Two conditions that ensure the instability of the disease-free equilibrium of the equations have been obtained, Equations (1.3) and (1.4). While the first one was previously obtained for the particular case of uncorrelated networks, as far as we know the second one is new. This last condition takes into account some of the probabilities involved in the conditional degree distribution and, hence, it is expected to give better estimations for the sufficient conditions of instability of the disease-free equilibrium, especially in the case of correlated networks.

In practice, both conditions imply the lack of an epidemic threshold for large networks with a fixed mean degree and nodes with large enough maximum connectivity. For mid-size networks, however, both conditions (1.3) and (1.4) give bounds for the critical values of the parameters (those that guarantee the instability of the disease-free equilibrium). We have investigated the goodness of the new condition with respect the classical one by studying the behavior of the bounds for the critical transmission rate in a number of networks with different degree distributions and a range of correlation coefficients. The results are in good agreement with the assertion that condition (1.4) gives a more accurate estimation of the epidemic threshold, especially for correlated networks.

\section{Acknowledgements}

Supported by Ministry of Economy and Competitiveness of the Spanish Government grant numbers MTM2011-27739-C04-03 (first author) and DPI2011-25822 (second author). Also by Generalitat de Catalunya projects 2009-SGR-345 (first author) and 2009-SGR-1228 (second author). 


\section{References}

[1] R.M. Anderson, R.M. May, Directly transmitted infectious-diseases: control by vaccination. Science 215 (1982), 1053-1060.

[2] A. Barabási, R. Albert, Emergence of scaling in random networks, Science 286 (1999), 509512.

[3] A. Berman, R.J. Plemmons, Nonnegative matrices in the Mathematical Sciences, Classics in Applied Mathematics 9, SIAM (1994).

[4] M. Boguñá, R. Pastor-Satorras, Epidemic spreading in correlated complex networks, Physical Review E 66 (4) (2002), 047104.

[5] V. Colizza, A. Vespignani, Invasion threshold in Heterogeneous metapopulation models, Physical Review Letters 99 (2007), 148701.

[6] V. Colizza, R. Pastor-Satorras, A. Vespignani, Reaction-diffusion processes and metapopulation models in heterogeneous networks, Nature Physics 3 (2007), 276-282.

[7] V. Colizza, A. Vespignani, Epidemic modeling in metapopulation systems with heterogeneous coupling pattern, J. theor. Biol. 251 (2008), 450-467.

[8] P. C. Cross, P. L. F. Johnson, J. O. Lloyd-Smith, W. M. Getz, Utility of $R_{0}$ as a predictor of disease invasion in structured populations, J. R. Soc. Interface 4 (2007), 4:315-24.

[9] K. Dietz, Overall population patterns in the transmission cycle of infectious disease agents, in Population Biology of Infectious Diseases (ed. R.M. Anderson, R.M. May), Dahlem Workshop Reports Vol. 25, pp 87-102. Springer (1982).

[10] K. T. D. Eames, M. J. Keeling, Modeling dynamic and network heterogeneities in the spread of sexually transmitted diseases, PNAS 99 (2002), pp. 13330-13335.

[11] H. Fukś, A.T. Lawniczak, R. Duchesne, Effects of population mixing on the spread of SIR epidemics, The European Physical Journal B, 50 issue 1 (2006), 209-214.

[12] J.L. Garcia-Domingo, D. Juher, J. Saldaña, Degree correlations in growing networks with deletion of nodes, Physica D 237 (2008), 640-651.

[13] R.A. Horn, C.R. Johnson, Matrix Analysis, Cambridge Univ. Press (1990).

[14] L. Hufnagel, D. Brockmann, T. Geisel, Forecast and control of epidemics in a globalized world, PNAS 101 (2004), 15124.

[15] D. Juher, J. Ripoll, J. Saldaña, Analysis and Monte Carlo simulations of a model for the spread of infectious diseases in heterogeneous metapopulations, Physical Review E 80 (2009), 041920. 
[16] M. J. Keeling, The effects of local spatial structure on epidemiological invasions, Proc. R. Soc. Lond. B 266 (1999), 859-869.

[17] H. McCallum, N. Barlow, J. Hone, How should pathogen transmission be modelled?, Trends in Ecology \& Evolution 16 (2001), 295-300.

[18] R. M. May, A. L. Lloyd, Infection dynamics on scale-free networks, Physical Review E 64 (2001), 066112.

[19] M. E. J. Newman, Spread of epidemic disease on networks, Physical Review E 66 (2002), 016128.

[20] M. E. J. Newman, Assortative mixing in networks, Phys. Rev. Lett. 89 (2002), 208701.

[21] Y.A. Rho, L. S. Liebovitch, I. B. Schwartz, Dynamical response of multi-patch, flux-based models to the input of infected people: Epidemic response to initiated events, Physics Letters A 372 (2008), 5017.

[22] J. Saldaña, Continuous-time formulation of reaction-diffusion processes on heterogeneous metapopulations, Physical Review E 78 (2008), 012902.

[23] J. Saldaña, Modelling the spread of infectious diseases in complex metapopulations, Mathematical Modelling of Natural Phenomema 5 (2010), No. 6, 22-37.

[24] H. R. Thieme, Mathematics in population biology, Princeton Univ. Press (2003).

[25] R. Xulvi-Brunet, I.M. Sokolov, Changing correlations in networks: assortativity and dissortativity, Acta Physica Polonica B 36 (2005), 1431-1455. 